\section{S9.4 INTERNATIONAL AIDS POLICY CHOICES FOR A CHANGING FINANCING LANDSCAPE}

doi:10.1136/sextrans-2011-050102.39

D Wilson. World Bank, Washington, District of Columbia, USA

Background In 2009, AIDS financing fell for the first time, reflecting the global financial crisis and shifting health and development priorities. In this context, future international AIDS policy must focus on efficiency, effectiveness and sustainability.

Efficiency The surge of financing for AIDS over the last decade has sometimes led to sub-optimal efficiency. There is an urgent need to improve allocative efficiency, by ensuring resources are allocated for the best proven and most relevant interventions among the right groups in the right geographic areas. AIDS responses globally are beset by mismatches between investments and epidemiological context and program choices. There is also a need to improve technical efficiency, so that selected interventions are delivered at scale, for the lowest cost. Several studies show how the same services in the same context in the same country can vary by several orders of magnitude. In a resource constrained future, such variances must be reduced and technical efficiency constantly improved. Integration of health-related HIV services and other health services may also yield greater efficiencies.

Effectiveness In concentrated epidemics, particularly among sex workers, where we have well validated interventions, our major priorities are to improve allocative and technical efficiency, so we direct our resources towards effective interventions for priority populations. In generalised epidemics, we need to strengthen our evidence of what works. Beyond male circumcision, we have limited proven interventions to reduce general population adult sexual transmission. We also need to move from efficacy to effectiveness, demonstrating that proven approaches can have real world effect at scale. We need to strengthen the middle-ground between randomised control trials and observation, by using quasi-experimental techniques to construct robust counterfactuals-that is, what would have happened without the counterfactual. We also need to make biological end-points, primarily HIV incidence or credible incidence proxy measures.

Sustainability We must move from an emergency response to longerterm sustainability. This will include increasing and diversifying domestic resource mobilisation, especially in middle-income countries and diversifying the sources of international financing. Services that are related to AIDS but also related to other elements of the health sector, such as blood safety and universal precautions will increasingly be financed from overall health sector resources.

Conclusion We must urgently re-position the global AIDS response, guided by the drive for greater efficiency, effectiveness and sustainability-we have no time to lose.

\section{Symposium 10: Strategic approaches for addressing sexual health: lessons. challenges and opportunities (sponsored by the CDC) S10.1 ADDRESSING SEXUAL HEALTH IN CANADA}

doi:10.1136/sextrans-2011-050102.40

L Smylie, B Clarke. Public Health Agency of Canada, Ottawa, Canada

Following a decline in reported rates of reportable sexually transmitted infections into the late-1990s in Canada, rates have been rising steadily with over two-thirds of reported chlamydia and gonorrhoea cases occurring among young people under 25 years of age. These increases have paradoxically occurred in the face of a decline in teenage pregnancy, an increase in condom use, a stable age of first sexual intercourse, and fewer lifetime sexual partners. Up to $35 \%$ of women and $15 \%$ of men report being coerced into sexual intercourse. Sexual minority adults experience higher rates of violent victimisation, including sexual assault, and rates of discrimination three times higher than heterosexuals. Sexual minority and gender variant individuals are up to seven times more likely to attempt suicide than heterosexuals. The burden of poor sexual health in Canada is unevenly distributed across the population with a concentration of poor sexual health outcomes among the economically disadvantaged, in more isolated areas, and among sexual minority and gender variant populations.

Our approach to addressing sexual health embraces a population health philosophy. The approach does not focus solely on sexual behaviour, but on determinants that influence the contexts within which decisions and choices affecting sexual health are made. It is a multi-sectoral approach fostering partnerships across government departments and with community organisations. It is a holistic approach that does not focus solely on the physical aspects of sexual health, but on the emotional, mental, and social aspects as well.

As part of a symposium on national approaches to sexual health, this presentation will examine key experiences, challenges and opportunities encountered in the Public Health Agency of Canada's approach to protecting and enhancing the sexual health of Canadians. In particular, it will highlight Public Health Agency of Canada's leadership and commitment to sexual health through its investment in research to develop national indicators of sexual health and to collect baseline national data; present exemplary health and social interventions to create supportive environments to promote the sexual health of all Canadians; and share innovative methods used to engage members of vulnerable populations in the development and implementation of sexual health interventions.

\section{S10.2 DEVELOPING AND IMPLEMENTING A PUBLIC HEALTH APPROACH TO IMPROVE SEXUAL HEALTH IN THE USA}

doi:10.1136/sextrans-2011-050102.41

J Douglas, K Fenton. Centers for Disease Control and Prevention, Atlanta, Georgia, USA

Background The first formal U.S. government recognition of the importance of a sexual health framework for enhancing STD/HIV prevention-The Surgeon General's Call to Action to Promote Sexual Health and Responsible Sexual Behaviour -was published in 2001. Since then, many measures of adverse health outcomes of sexual behaviour have improved only minimally or worsened, including HIV, STD, and teen pregnancy. CDC is committed to enhancing program impact for STD/HIV prevention and other programs by complementing traditional prevention efforts with a health promotion framework that comprehensively addresses the broader issue of sexual health.

Methods We have developed a cross-CDC Sexual Health Steering Committee including Divisions of STD Prevention, HIV/AIDS Prevention, Viral Hepatitis, Adolescent/School Health, Reproductive Health, and Violence Prevention which works collaboratively with a Sexual Health Workgroup of the CDC/HRSA Advisory Committee on HIV and STD.

Results Key objectives of the CDC sexual health effort include increased knowledge and awareness of and healthy/respectful attitudes regarding sexual health; increased use of high-quality, coordinated and integrated educational, programmatic, and clinical services; increased healthy, responsible, and respectful sexual behaviours; improved healthy and respectful sexual relationships, free of coercion; and decreased adverse health outcomes, including HIV/STD, viral hepatitis, unplanned pregnancy, and sexual and intimate partner violence. Initial efforts focused on a policy discussion paper and expert consultation on "A Public Health 
Approach for Advancing Sexual Health in the USA" Subsequent steps have included expanding engagement of stakeholders from a broad range of perspectives; steps to evaluate a sexual health communication framework; an expanded CDC sexual health website; efforts to incorporate sexual health into key U.S. policy documents (eg, National HIV AIDS Strategy, Healthy People 2020 key indicators; National Prevention Strategy); planning a national coalition to address sexual health in the general population, adolescents/young adults, and among MSM; and development of a conceptual framework, logic model, and CDC definition of sexual health to inform a final white paper on sexual health.

Conclusions Developing and implementing a sexual health framework in a society with diverse values is challenging, but core public health prevention efforts must be enhanced by a renewed effort to address issues of sexual health and responsible sexual behaviour as an intrinsic part of human health. The use of a sexual health framework has great potential for improving public health outcomes by providing messages that are more effective at reaching the public and providers, enhancing the efficiency and effectiveness of services, and facilitating societal dialogue around sexuality and sexual behaviour.

\section{S10.3 SEXUAL HEALTH IN MSM-COORDINATION AND AGENDA SETTING IN EUROPE}

doi:10.1136/sextrans-2011-050102.42

M Van de Laar. European Centre for Disease Prevention and Control, Stockholm, Sweden

The European Centre for Disease Prevention and Control (ECDC) has a strong mandate with respect to surveillance of communicable disease in the European Union but a less strong mandate regarding prevention and control as this remains the responsibility of individual Member States. However, ECDC has produced several guidance documents and user-friendly toolkits to support public health decision makers and experts in the Member States to strengthen available prevention and control activities.

In the light of (1) increasing trends of STI and the continuous rise in the number of newly diagnosed HIV infections among MSM and (2) the evidence for increased high-risk sexual behaviour and the widespread sexual mixing among MSM in Western Europe, ECDC has assessed effectiveness of behavioural and psychosocial HIV/STI prevention interventions for MSM in Europe and concluded that there is an overall deficit in outcome evaluations of interventions aimed at reducing HIV/STI risk behaviour among MSM in Europe.

Following this background, ECDC has launched an additional project to include a review and assessment of the following: interventions aiming at knowledge, attitudes and beliefs and influencing psychological and social risk correlates (eg, media campaigns, interpersonal education programmes, sexual health education, safer sex promotion, detached education, outreach work, prevention counselling, peer education, community level interventions). Interventions aiming at lowering the risk of behaviour (eg, condom distribution, provision of paraphernalia for safer sex) The final report contains a compilation and summary of publications regarding outbreaks and increasing trends of HIV and STI, an update of different prevention interventions targeted at MSM as well of interventions planned and carried out within the framework of programmatic responses to the HIV epidemic as well as interventions following outbreaks or other events, a review of evaluation of different interventions and synthesis the evidence base for preventive interventions, and a synopsis of identified knowledge gaps.

Based on the review and the assessment, a platform for multidisciplinary expert consultation will be organised in August 2011 (including epidemiologists, prevention experts, social behaviour scientists, representatives of civil society, Member States, NGOs, and other key stakeholders) to discuss the needs and challenges for coordination at EU level.

\section{S10.4 SEXUAL HEALTH AND PUBLIC POLICY IN LATIN AMERICA AND THE CARIBBEAN (LAC)}

doi:10.1136/sextrans-2011-050102.43

R Mazin. Pan American Health Organization/Regional Office of the World Health Organization for the Americas, Washington, District of Columbia, USA

Background Sexual health and its determinants are central to overall well-being and development. Thus, sexual health should be considered by public health as a critical element not only of the population's health, but also as an essential component of the health generated by the population. Regrettably, strict social norms and traditions in LAC have maintained the public discussions about sexuality in secretive and private domains. Moreover, rigid and stereotyped gender roles and social mores and values regarding sexuality have limited the incorporation of this area in the public health agendas, with the only exception of issues related to reproduction.

Methods With the purpose to initiate a process aimed at incorporated the notion of sexual health into the public agenda, in May 2000, the Pan American Health Organization, in association with the World Association for Sexology/WAS (which has since changed its name to World Association for Sexual Health), held a consultation in Guatemala in which a definition of sexual health was coined: a state of physical, psychological, and socio-cultural well-being related to sexuality. The sexual health and the quality of sexual life of individuals, families and communities and the various factors that can affect them were also discussed and avenues for action were proposed.

Since then PAHO has also been engaged in several processes to further advance the sexual health agenda in the Americas, among which the collaboration with WAS in their initiative "Sexual Health for the Millennium", the explicit incorporation of indicators on sexual health in PAHO's Strategic Plan for the Region, active support to the Mexico City Declaration, and several others intended to improve the health of members of sexual minorities and to rekindling of STI programs in LAC.

Results Preliminary empirical evidence seems to indicate that the use of a public health rationale contributes to create convergence in an open debate that would be impossible to hold if other approaches were to be used (eg, based on moral, tradition or assumptions and beliefs). The discussion should lead to the realisation of sexual health, which means adoption of healthy lifestyles and the creation of supportive environments conducive to well-being for all. It would also contribute to the reorientation of the health services to better respond to the sexual needs of individuals through the life cycle, families, and communities.

\section{Symposium 11: Controversies in serologic testing for syphilis (sponsored by the CDC) S11.1 PROBLEMS ENCOUNTERED WITH REVERSE SEQUENCE SYPHILIS SCREENING}

doi:10.1136/sextrans-2011-050102.44

K W Hoover. Centers for Disease Control and Prevention, Atlanta, Georgia, USA

CDC has long recommended screening for syphilis with a nontreponemal test such as the RPR test and, if reactive, confirming with a treponemal test. Non-treponemal antibody levels rise during 